\title{
छs \\ Matching-based fresh-slice method for generating two-color x-ray free-electron lasers
}

\author{
Weilun Qin, ${ }^{1,2}$ Yuantao Ding, ${ }^{2}$ Alberto A. Lutman, ${ }^{2}$ and Yu-Chiu Chao,* \\ ${ }^{1}$ Institute of Heavy Ion Physics, School of Physics, Peking University, Beijing 100871, China \\ ${ }^{2}$ SLAC National Accelerator Laboratory, Menlo Park, California 94025, USA
}

(Received 24 May 2017; published 5 September 2017)

\begin{abstract}
Two-color high intensity $\mathrm{x}$-ray free-electron lasers (FELs) provide powerful tools for probing ultrafast dynamic systems. A novel concept of realizing fresh-slice two-color lasing through slice-dependent transverse mismatch has been proposed by one of the authors [Y. Chao, SLAC Report No. SLAC-PUB-16935, 2016]. In this paper we present a feasible example following this concept based on the Linac Coherent Light Source parameters. Time-dependent mismatch along the bunch is generated by a passive dechirper module and controlled by downstream matching sections, enabling FEL lasing at different wavelength with a split undulator configuration. Simulations for soft x-ray FELs show that tens of gigawatts pulses with femtosecond duration can be generated.
\end{abstract}

DOI: 10.1103/PhysRevAccelBeams.20.090701

\section{INTRODUCTION}

High-brightness, short-duration x-ray free-electron lasers (XFELs), such as the Linac Coherent Light Source (LCLS) [1], the SPring-8 Angstrom Compact Free-Electron Laser (SACLA) [2], the European XFEL [3] and so on, open up a wide range of applications in probing both the ultrasmall scale and ultrafast processes. Advanced operational modes of the XFELs have been developed to meet specific requirements of various scientific applications. Within these advanced modes, the twocolor XFEL, delivering two synchronized pulses with controllable time delay and wavelength separation, has been an intense subject of study, enabling pump-probe experiments capturing dynamical processes of chemical reactions and electronic evolution in materials on the atomic scale $[4,5]$.

The wavelength of the XFEL radiation emitted is related to the undulator parameters and the electron bunch energy as

$$
\lambda_{r}=\frac{\lambda_{u}}{2 \gamma^{2}}\left(1+\frac{K^{2}}{2}\right)
$$

where $\lambda_{u}$ is the undulator period, $K$ is the undulator strength parameter and $\gamma$ is the Lorentz factor. Two-color radiation is usually enabled by using two different undulator strengths or electron beam energies. Two-color XFEL was first demonstrated at the LCLS in self-amplified spontaneous emission (SASE) mode using a split undulator scheme [6]. The delay

\footnotetext{
*ychao@slac.stanford.edu
}

Published by the American Physical Society under the terms of the Creative Commons Attribution 4.0 International license. Further distribution of this work must maintain attribution to the author(s) and the published article's title, journal citation, and DOI. between the two-color pulses was controlled by a magnetic chicane between the two undulator sections. Large wavelength separation was demonstrated using variable gap undulators [7]. A twin-bunch scheme used two electron bunches, generated by pulse stacking at the photo-injector, to generate two-color pulses with high intensity [8]. With various seeding methods, the spectral purity of the twocolor pulses can be improved at different photon energies [9-12]. Note there are also studies concerning period tunable undulators $[13,14]$, which can enrich the schemes to generate two-color FELs.

Recently, a fresh-slice method generating multicolor, fully saturated $x$-ray pulses has been demonstrated at the LCLS [15]. This method relies on an electron bunch tailored with time-dependent transverse kick effect introduced by passing the electron bunch offaxis in a dechirper device [16-18] before entering the undulator. Electron temporal slices at the bunch head stay on-axis since the dipole kick of the dechirper is negligible at the bunch head, while electron slices at the bunch tail travel along trajectories with large orbit oscillations. Steering either the bunch head or the tail to the on-axis orbit in the first undulator section enables the on-axis slices to lase, while the slices with large orbit oscillations are still fresh since the lasing is suppressed. In the second undulator section the fresh-slices are steered back to the on-axis orbit and then lase at the second color.

Instead of using the dipole effect which causes a large orbit distortion downstream of the dechirper, one of the authors (Y. Chao) has proposed using the time-dependent quadrupole effect of the dechirper device to generate freshslice lasing based two-color x-ray pulses [19]. When the electron bunch traverses the dechirper, temporal slices experience a time-dependent quadrupole effect, resulting in progressively mismatched slices from head to tail. This effect can be relevant also when the electron bunch travels 


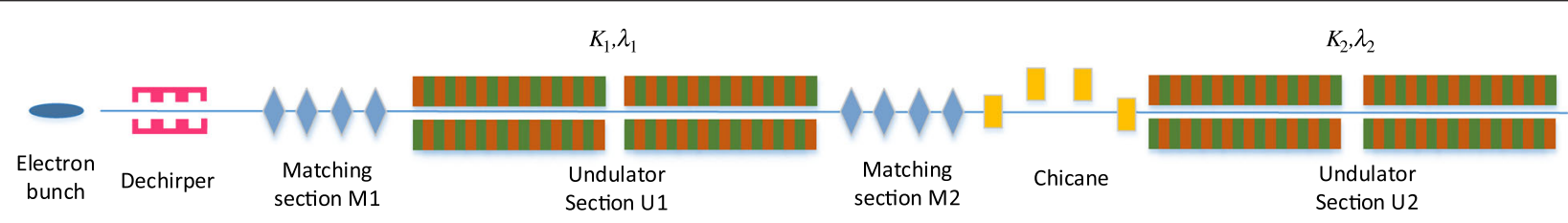

FIG. 1. Schematic of the layout to generate two-color FEL pulses. The dechirper device generates a time-dependent mismatch along the temporal slices, with bunch head still matched while bunch tail is mismatched by a large factor. The first matching section (M1) matches the bunch tail and mismatches the bunch head. The first color x-ray pulse is generated at the bunch tail in the first undulator section (U1) tuned to $K_{1}$. Another matching section (M2) is placed after U1 to match the bunch head. The beam is then sent into the second undulator section (U2) tuned to $K_{2}$ to generate the second color X-ray pulse. A magnetic chicane can be used to control the time delay of the two-color pulses.

on the dechirper axis. In the undulator, lasing is suppressed to a different degree for each slice according to its mismatch to the undulator lattice. Adopting the split undulator configuration, one can use a matching section to select the bunch head or tail to lase at one color in the first undulator section. Subsequently, another matching section is used to match the formerly mismatched slices at the entrance to the second undulator section. The rematched slices lase at a second color in the second undulator section. Since there is no bunch orbit distortion for this method, it can greatly ease the charge loss problem along the transport beamline and the undulator [20], potentially being a better solution for high repetition-rate XFELs. It is also beneficial to combine both the dipole component and the quadrupole component of the dechirper to reduce the orbit distortion in low repetition-rate XFELs.

The layout of the proposed scheme is described in Sec. II, followed by the generation of a time-dependent mismatch of the electron bunch using a dechirper in Sec. III. In Sec. IV, we describe how a dedicated matching section can be used to select the lasing slice. We present two-color FEL simulations in Sec. V. The beam tracking and FEL simulations are performed using typical LCLS soft $\mathrm{x}$-ray operating parameters. We also discuss the implementation and application of this method. Results are summarized in Sec. VI.

\section{LAYOUT}

The layout of the proposed method is sketched in Fig. 1. A dechirper device (see Fig. 2) is located in the upstream of the undulator line. After passing through the dechirper on
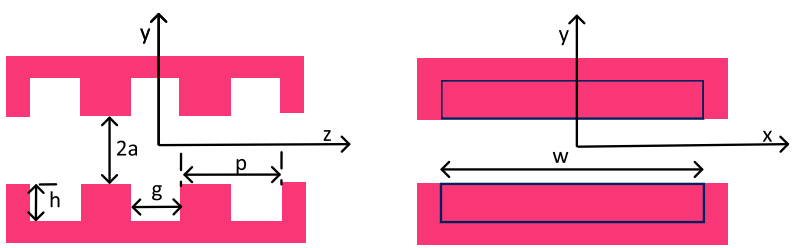

FIG. 2. Structure of the vertical dechirper device, which is composed of two parallel metallic jaws with corrugations. The half-gap of the dechirper is $a$, period is $p$, opening is $g$, depth is $h$, and width is $w$. The electron bunch traverses in the $z$ direction. axis, the bunch tail is significantly mismatched with respect to the design lattice due to the time-dependent quadrupole effect of the dechirper wakefields, while the head part stays matched. A four-quad matching section (M1) is used to match the tail and mismatch the head at the same time so that the tail part can lase in the first undulator section (U1) tuned to $K_{1}$. Another four-quad matching section (M2) located between the two undulator sections is used to match the head part and mismatch the tail so that the head part now can lase in the second undulator (U2) at wavelength $\lambda_{2}$. Besides the large mismatch, the energy spread of the tail is increased in $\mathrm{U} 1$ after lasing, making the suppression of the tail in U2 more effective. A chicane between the two undulator sections can be used to adjust the time delay of the two-color pulses and also wash out the microbunching formed in the first undulator section at the bunch tail. Note that we choose the bunch tail to lase first so that we have more flexibility to control of delay of the two pulses.

The existing LCLS parameters are used to demonstrate the feasibility of the proposed scheme. The main machine parameters used in simulations are listed in Table I. The dechirper system, including one 2-m long vertical dechirper

TABLE I. Main parameters used in simulations.

\begin{tabular}{lcc}
\hline \hline Electron and radiation & Value & Units \\
\hline Energy $E$ & 4.4 & $\mathrm{GeV}$ \\
Bunch charge $Q$ & 180 & $\mathrm{pC}$ \\
Bunch length $\ell$ & 70 & $\mathrm{fs}$ \\
Emittance & 0.5 & $\mu \mathrm{m}$ \\
Current & 2.5 & $\mathrm{kA}$ \\
Twiss $\beta_{x} / \beta_{y}$ before dechirper & $4.49 / 100$ & $\mathrm{~m}$ \\
Twiss $\alpha_{x} / \alpha_{y}$ before dechirper & $0.02 / 3.04$ & \\
Undulator period $\lambda_{u}$ & 3 & $\mathrm{~cm}$ \\
Undulator K at U1/U2 & $3.45 / 3.50$ & \\
Wavelength $\lambda_{r}$ at U1/U2 & $1.41 / 1.45$ & $\mathrm{~nm}$ \\
Dechirper vertical module & & \\
Half-gap $a$ & 0.75 & $\mathrm{~mm}$ \\
Period $p$ & 0.5 & $\mathrm{~mm}$ \\
Depth $h$ & 0.5 & $\mathrm{~mm}$ \\
Opening $g$ & 0.25 & $\mathrm{~mm}$ \\
Width $w$ & 12.7 & $\mathrm{~mm}$ \\
Length $L$ & 2 & $\mathrm{~m}$ \\
\hline \hline
\end{tabular}


module and one 2-m long horizontal dechirper module, is installed in the transport beamline from linac to undulator (LTU). Since the time-dependent quadrupole effect of the vertical dechirper can be compensated by the horizontal dechirper, we only use the vertical dechirper, and leave the horizontal dechirper jaws fully retracted. One dedicated matching section (M1) which already exists after the dechirper can be used to select the first lasing slice. In principle, the second matching section (M2) to switch the lasing slice can be realized using existing quadrupoles between undulator segments, but the quadrupole strength at the LCLS is not sufficient to match the largely mismatched slice back. Thus we consider a dedicated matching section with four quadrupoles replacing one existing undulator segment. The overall length of this matching section is about $4.8 \mathrm{~m}$ taking into account the existing drift space on both ends of the undulator segments. The lengths of the quadrupoles are $0.3 \mathrm{~m}$, the same with the existing undulator quadrupoles, and the gradients are on the same level of the existing matching section M1. The LCLS planar undulator segments have fixed gap, but the canted angle of the magnetic poles allows for tuning $K$ between 3.44 and 3.51 . Two magnetic chicanes, replacing the 9th and the 16th undulator segments for soft x-ray [21] and hard x-ray selfseeding [22], can be used to provide delay control between the two-color pulses. The second matching section (M2) can be located between the two magnetic chicanes. In the simulation presented in this paper, M2 is at the 15th undulator segment prior to the hard x-ray self-seeding chicane.

\section{GENERATION OF TIME-DEPENDENT MISMATCH USING A DECHIRPER}

The geometry of the vertical dechirper device is sketched in Fig. 2, with half-gap $a$, period $p$, opening $g$, depth $h$, and width $w$. The electron bunch traverses in the $z$ direction. The dechirper parameters used are also listed in Table I.

In a vertical flat dechirper, both dipole wake $w_{y d}$ and quadrupole wake $w_{y q}$ exist. The total transverse wake effect of the vertical dechirper near the axis can be given by

$$
\begin{aligned}
& w_{y}=y_{0} w_{y d}+y w_{y q}, \\
& w_{x}=x_{0} w_{y q}-x w_{y q},
\end{aligned}
$$

where $\left(x_{0}, y_{0}\right)$ and $(x, y)$ are the offset of the driving particles and test particles, respectively. The dipole wake $w_{y d}$ and quadrupole wake $w_{y q}$ are given by [23]

$$
\begin{aligned}
& w_{y d}(s) \approx \frac{\pi^{4}}{16 a^{4}} s_{0 d}\left[1-\left(1+\sqrt{\frac{s}{s_{0 d}}}\right) e^{-\sqrt{s / s_{0 d}}}\right] \\
& w_{y q}(s) \approx \frac{\pi^{4}}{16 a^{4}} s_{0 q}\left[1-\left(1+\sqrt{\frac{s}{s_{0 q}}}\right) e^{-\sqrt{s / s_{0 q}}}\right]
\end{aligned}
$$

with

$$
s_{0 d}=s_{0 r}\left(\frac{15}{14}\right)^{2}, \quad s_{0 q}=s_{0 r}\left(\frac{15}{16}\right)^{2} .
$$

Here $s_{0 r}=\frac{a^{2} g}{2 \pi \alpha^{2} p^{2}}$, and $\alpha(\kappa) \approx 1-0.465 \sqrt{\kappa}-0.070 \kappa$ with $\kappa=g / p$.

The short-range bunch wake is given by a convolution of the point charge wake function $w(s)$ and bunch longitudinal charge distribution $\lambda(s)$,

$$
W_{\lambda}(s)=\int_{0}^{\infty} d s^{\prime} w\left(s^{\prime}\right) \lambda\left(s-s^{\prime}\right) .
$$

For an electron beam with symmetric transverse charge distribution, dipole kick due to wakefields vanishes when the beam traverses on-axis of the dechirper. For a uniform longitudinal distribution with full bunch length $\ell$, the quadrupole bunch wake is given in the form of

$W_{\lambda q}(s)=\frac{\pi^{4} s_{0 q}}{16 a^{4} \ell}\left[2 e^{-\sqrt{\frac{s}{s_{0 q}}}}\left(3 \sqrt{s s_{0 q}}+s+3 s_{0 q}\right)+s-6 s_{0 q}\right]$.

The inverse focal length of the quadrupole bunch wake is given by

$$
f_{q}^{-1}(s)=\mp\left(\frac{Z_{0} c}{4 \pi}\right) \frac{e Q L W_{\lambda q}(s)}{E},
$$

where $Z_{0}=377 \Omega$ is the vacuum impedance, $c$ is the speed of light in vacuum, $e$ is the electronic charge, $Q$ is bunch charge, $L$ is dechirper length, and $E$ is the beam energy. The minus and plus signs represent the inverse focal length for $x$ and $y$, respectively. This indicates that the vertical dechirper is focusing in $x$ and defocusing in $y$. The quadrupole wake effect can be expressed in matrix form as

$$
M_{q}(s)=\left[\begin{array}{cccc}
1 & 0 & 0 & 0 \\
-\frac{1}{\left|f_{q}(s)\right|} & 1 & 0 & 0 \\
0 & 0 & 1 & 0 \\
0 & 0 & \frac{1}{\left|f_{q}(s)\right|} & 1
\end{array}\right] .
$$

Note that this quadrupole effect is related to bunch internal coordinate $s$, thus bunch head and tail experience different focusing when traveling through the dechirper. For the typical LCLS short bunches, with a duration of tens of femtoseconds and uniform current, the quadrupole effect is with good approximation quadratic. The time-dependent focusing yields a time-dependent mismatch along the temporal slices.

To extend the widely used 2D betatron mismatch factor Bmag [24], we define $\Phi$ as the 4D mismatch factor 
between the electron bunch and the lattice [19], after a transport of $M$,

$$
\Phi=\frac{1}{4} \operatorname{Trace}\left[\Sigma_{d}^{-1}\left(M \Sigma_{b} M^{T}\right)\right]
$$

where $\Sigma$ is the normalized beam covariance matrix and takes on the form below in the absence of XY coupling

$$
\Sigma=\left[\begin{array}{cccc}
\beta_{x} & -\alpha_{x} & 0 & 0 \\
-\alpha_{x} & \gamma_{x} & 0 & 0 \\
0 & 0 & \beta_{y} & -\alpha_{y} \\
0 & 0 & -\alpha_{y} & \gamma_{y}
\end{array}\right],
$$

with subscript $d$ representing the design lattice Twiss at the end of the transport, and subscript $b$ representing the bunch Twiss entering the transport. By inserting the Twiss and dechirper matrices into Eq. (11), one can obtain the mismatch along the bunch.

It is important to note that the mismatch produced at the bunch tail is related to the incoming Twiss parameters, more specifically, the average beam size through the dechirper. To generate mismatch as large as possible, it is favorable to increase the average beam size. To avoid hitting on the dechirper wall, the slice with largest size along the bunch should be well within the dechirper gap. Using LCLS parameters as listed in Table I, we plot the mismatch at the bunch tail as a function of incoming $\beta_{y}$ and $\alpha_{y}$ in Fig. 3. The white dashed lines show the beta values of bunch tail $\beta_{\text {tail }}$ at the dechirper exit. Colored contours represent $\Phi_{\text {tail }}$ with respect to the design Twiss. It is seen that for the same mismatch value, launching a focusing beam into the dechirper is beneficial to control the beam size in the dechirper. In the following, we choose

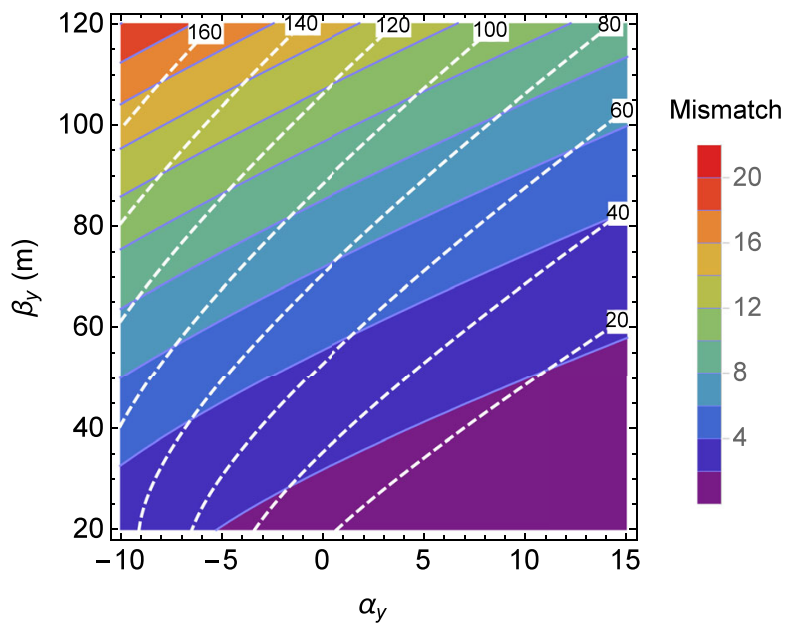

FIG. 3. Colored contours show mismatch of bunch tail at the dechirper exit as a function of incoming Twiss parameters. White dashed lines represent the beta values of the bunch tail at the dechirper exit. Bunch head is matched to the lattice.
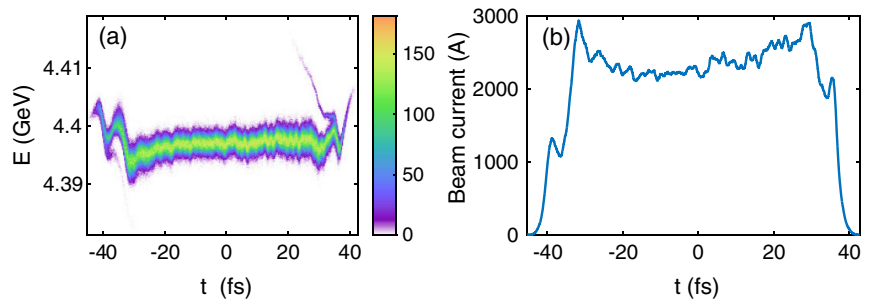

FIG. 4. Electron bunch longitudinal phase space (a) and current profile (b) at the entrance of the undulator with dechirper inserted. Bunch head is on the left. The relatively flat current profile is obtained using a collimation mode at the LCLS.

$\beta_{y}=100 \mathrm{~m}, \alpha_{y}=3$ before the dechirper, with estimated mismatch value 9.5. The corresponding largest slice (rms) beam size is $66 \mu \mathrm{m}$, sufficiently small compared to the $1.5 \mathrm{~mm}$ dechirper gap. The Twiss in $x$ is set to the design value as $\left(\beta_{x}, \alpha_{x}\right)=(4.49 \mathrm{~m}, 0.02)$, which causes negligible mismatch along the temporal slices in $x$.

In a start-to-end simulation, we tracked an LCLS bunch from the dechirper to the undulator entrance using ELEGANT [25]. The electron bunch current profile was shaped to be relatively flat using a collimation mode developed at the LCLS [26]. The electron bunch energy chirp was cancelled by the longitudinal wakefield of the dechirper. The longitudinal phase space and current profile are shown in Fig. 4. The dechirper transverse wakefields are implemented in ELEGANT using the TRWAKE element, and providing the point charge wake functions given in Eqs. (4) and (5). In Fig. 5, we show the transverse phase space distributions $t-x, t-x^{\prime}, t-y$, and $t-y^{\prime}$ at the entrance of the undulator, for the case the dechirper is retracted (a,c,e,g) and inserted (b,d,f,h). Bunch head is on the left. Phase space with the dechirper retracted shows the design pretty uniform beam size and beam divergence along the bunch. With the dechirper inserted, the influences of the dechirper quadrupole wakefield on $x$ and $x^{\prime}$ are negligible. However, there is a significant beam divergence increase in $y^{\prime}$ toward the bunch tail. The beam size in $y$ at the tail is also increased compared to when the dechirper is retracted but the variation is smaller than that in $t-y^{\prime}$ phase space. The variation in phase space may change depending on the initial Twiss parameters at the dechirper and betatron oscillation phase at the observation location in the beam line. We calculated the mismatch with respect to the design lattice Twiss for different temporal slices, as is shown in Fig. 6. When the dechirper is retracted, the projected Twiss parameters of the bunch are matched to the design values. The bunch head and tail are slightly mismatched due to collective effects (e.g., coherent synchrotron radiation) upstream of the dechirper [27]. When the dechirper is inserted, a progressive mismatch from the bunch head to tail is introduced, which depends approximately quadratically on time, $t$, as is shown in Fig. 6(b). The mismatch factors along the temporal slices remain constant during 

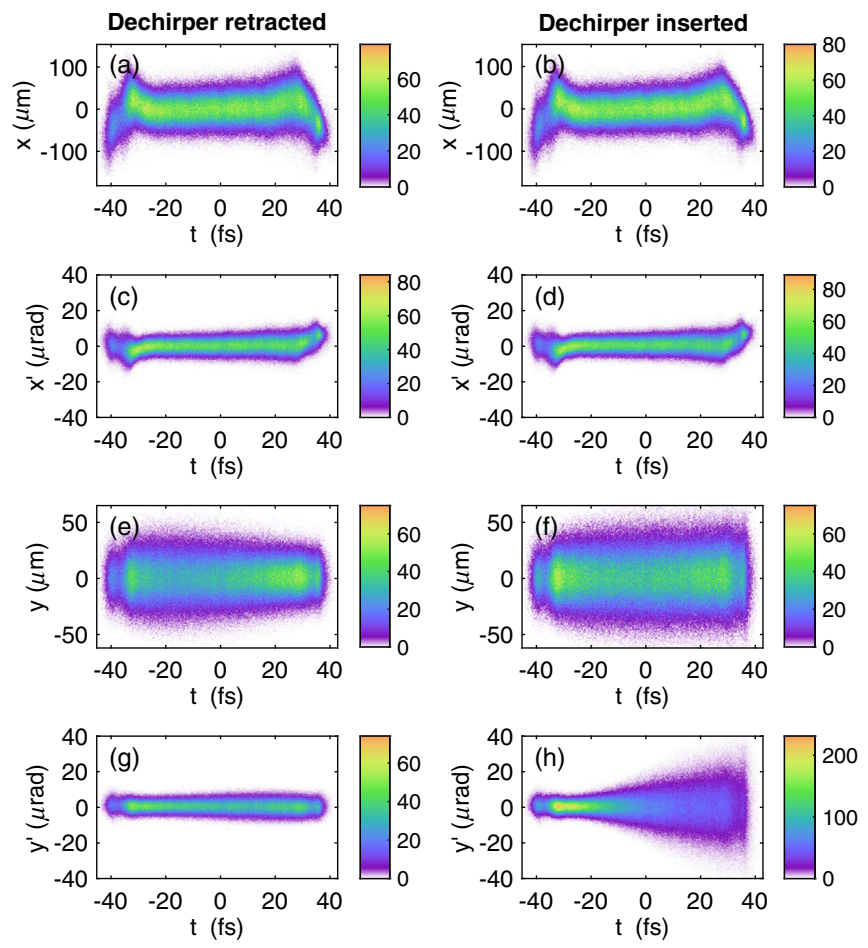

FIG. 5. Phase space distributions as function of time at the entrance of the LCLS undulator when the dechirper is retracted (a,c,e,g) and inserted (b,d,f,h). Bunch head is on the left. The influences of the dechirper quadrupole wakefield on $x$ and $x^{\prime}$ are negligible. There is a large beam divergence variation along the bunch in $y^{\prime}$ when the dechirper is inserted, while the variation in beamsize is relatively small. Due to the betatron oscillations in the transport beamline, there is an exchange in the variation between $y$ and $y^{\prime}$, but the relative mismatch with respect to the lattice is preserved for each temporal slice.
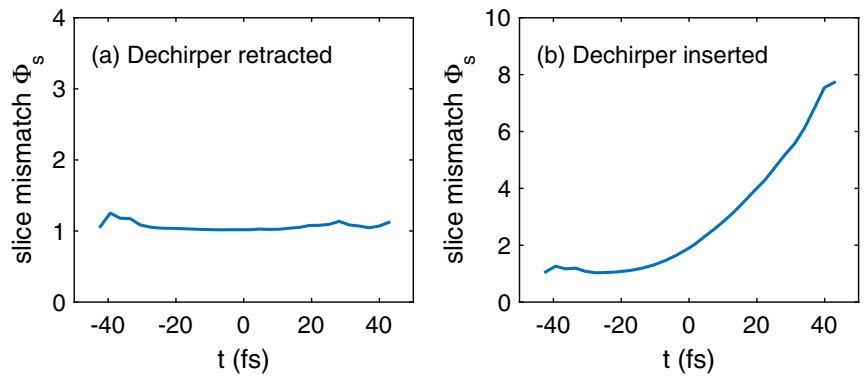

FIG. 6. Mismatch for different temporal slices with respect to design Twiss when the dechirper is retracted (a) and inserted (b). Bunch head is on the left.

transport. The introduced mismatch reaches a maximum of about 8 at the bunch tail, which is slightly smaller than the one calculated for an ideal flat-top current.

\section{FRESH-SLICE MATCHING}

One direct application of the time-dependent mismatch is to control the FEL pulse duration by adjusting the

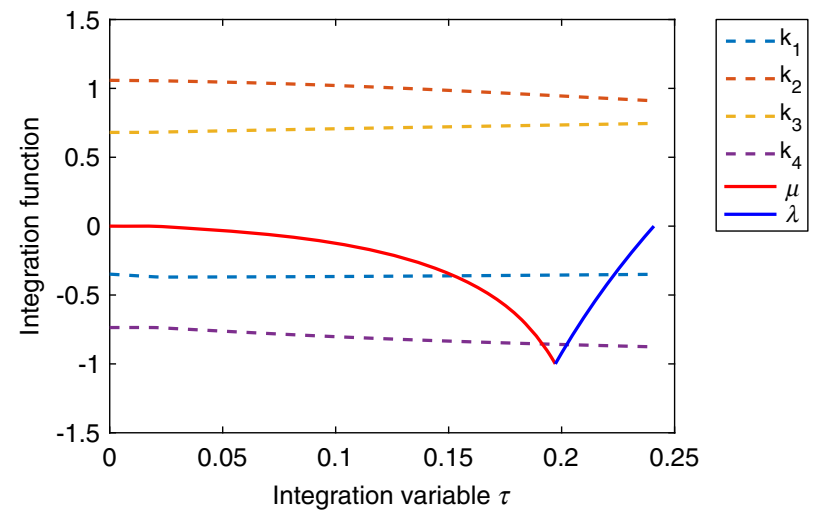

FIG. 7. Integration results showing the evolution of the quadrupole strengths and Lagrange multipliers along the integration variable. The integration starts from $\mu=0$ to $\mu=-1$ and then switches to integrate from $\lambda=1 / \mu=-1$ to $\lambda=0$.

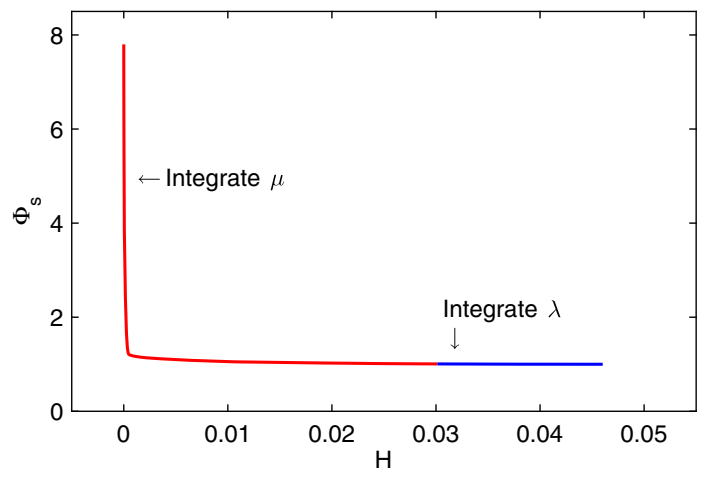

FIG. 8. Tradeoff curve between mismatch $\Phi_{s}$ and rms deviation of quadrupole strength. By integrating using the deterministic method, the 4-quadrupole system moves from the mismatched end to the matched end.

electron bunch Twiss parameters at the dechirper entrance or the dechirper gap. To enable more elaborate schemes such as two-color generation, it is necessary to provide flexible control of the matched position along the bunch. A matching section consisting of quadrupoles should be used. For a matching section with $N_{q}$ quadrupoles, the 4D mismatch $\Phi_{s}$ for a temporal slice at the end of the matching section is a function of the quadrupole strengths

$$
\Phi_{s}\left(k_{1}, k_{2}, k_{3}, \ldots, k_{N_{q}}\right)=\frac{1}{4} \operatorname{Trace}\left[\Sigma_{d}^{-1}\left(M \Sigma_{s} M^{T}\right)\right],
$$

where $\Sigma_{s}$ is the Twiss matrix for the mismatched slice and $M$ is the transport matrix of the matching section. The solution of $\Phi_{s}=1$ gives the quadrupole strength combination that can match back the mismatched slice.

Many algorithms can be used to solve the matching problem. In this study, we adopt a deterministic matching algorithm [19]. In this method, a competing objective function $H$ against the mismatch $\Phi_{s}$ is defined via 

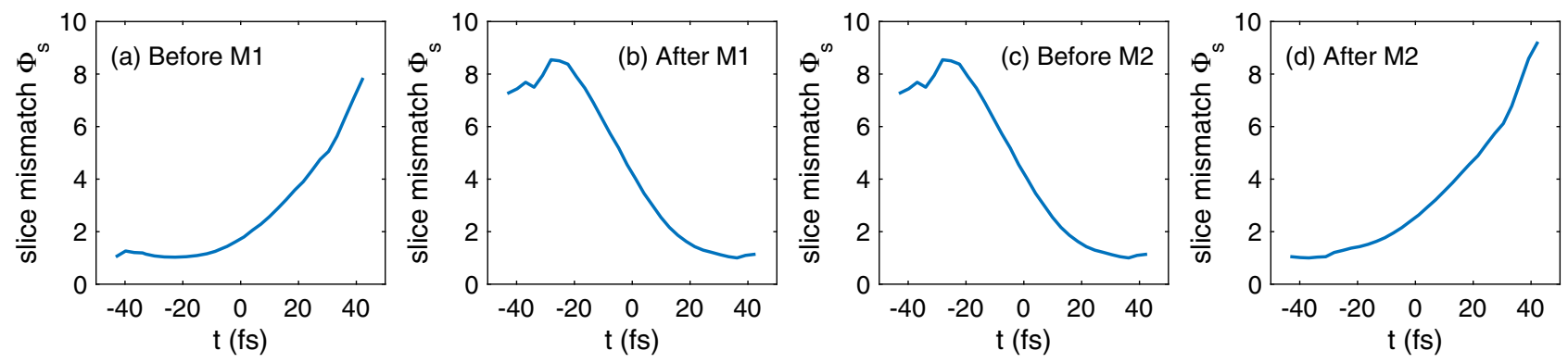

FIG. 9. Slice mismatch before and after matching section M1 and M2. The bunch head is on the left. The bunch tail is matched to the design lattice after M1 and bunch head is mismatched. The bunch head is matched back after M2.

$$
H=\Delta K=\Sigma_{i=1}^{N_{q}}\left(k_{i}-k_{i 0}\right)^{2}
$$

where $k_{i 0}$ are the initial values of the quadrupole strengths. The optimal tradeoff between $\Phi_{s}$ and $H$ follows a onedimensional tradeoff curve in the form of

$$
\begin{aligned}
& \nabla \Phi_{s}=\lambda \nabla H, \\
& \nabla H=\mu \nabla \Phi_{s},
\end{aligned}
$$

where $\lambda$ and $\mu$ are the Lagrange multipliers. Equation (16) is the conjugate form of Eq. (15) and $\mu=1 / \lambda$. The tradeoff curve connects the local optimal of $\Phi_{s}$ and $H$ on both ends. Starting with the initial condition of quadrupoles corresponding to $\nabla H=0, \lambda=-\infty, \mu=0$, one can use a numerical integration algorithm to integrate along the tradeoff curve until $\nabla \Phi_{s}=0, \lambda=0, \mu=-\infty$, where the minimal of mismatch is achieved. The integration starts
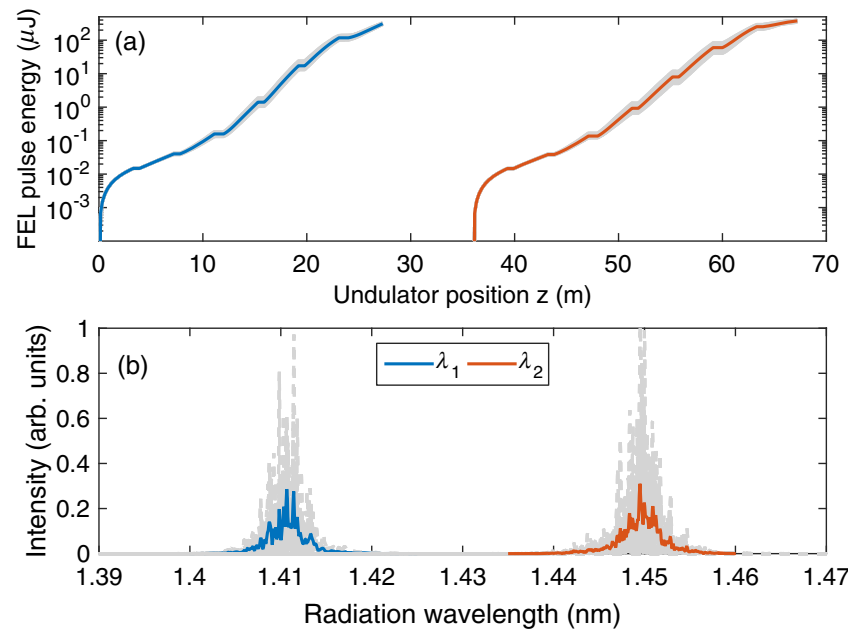

FIG. 10. Multiple-shots FEL pulse energy vs undulator position (a) and corresponding final spectra (b) for the two-color pulses. The grey lines represent the results of each individual shot and the colored lines represent the average over the 10 shots. The first color at $1.41 \mathrm{~nm}$ wavelength reaches pulse energy of $310 \pm 13 \mu \mathrm{J}$ after 7 undulator segments. The second color at $1.45 \mathrm{~nm}$ wavelength reaches pulse energy of $377 \pm 17 \mu \mathrm{J}$ after 8 undulator segments. from $\mu=0$ and continues until $\mu=-1$, and then switches to integrate $\lambda$ from -1 to 0 . With such an integration routine, an infinity of $\lambda$ at the initial point and of $\mu$ at the endpoint are avoided.

In the proposed two-color scheme, there are two matching sections, each consisting of 4 quadrupoles. We use the first matching section M1 to match the bunch tail to the design values so that the bunch tail can lase at the first color in U1. Then we use the second matching section M2 to match the bunch head and enable lasing at the second color in U2. Figure 7 shows the evolution of the quadrupole strengths and parameters $\lambda$ and $\mu$ via integration along an implicit variable $\tau$ for M1 matching. At the end of the integration where $\lambda=0$, the mismatched slice is matched again. Figure 8 shows the tradeoff curve between mismatch $\Phi_{s}$ and rms deviation of quadrupole strength $H$. By integrating using the deterministic method, the 4-quadrupole system moves from the tail mismatched end to the tail matched end of the tradeoff curve. Figure 7 also shows how the quadrupole strengths can be smoothly tuned to match a slice. The bunch slice mismatch before and after the two matching sections are shown in Fig. 9. The bunch tail is matched after the first matching section and the bunch head is matched back after the second matching section.

\section{FEL SIMULATIONS}

Using the electron bunch with bunch tail matched while head mismatched [Fig. 9(b)], we performed 10 shots of GENESIS [28] simulations using the LCLS undulator line. The two undulator sections consist of 7 and 8 undulator segments, respectively. The undulator parameters of the two sections are $K_{1}=3.45$ and $K_{2}=3.50$, which correspond to radiation wavelengths of $\lambda_{1}=1.41 \mathrm{~nm}$ and $\lambda_{2}=1.45 \mathrm{~nm}$. The strength of the chicane is set to $R_{56}=0 \mu \mathrm{m}$. There is no additional delay between the two pulses in this setup, but due to the slippage effect and lasing at different slices of the bunch, a time separation appears.

Figure 10 shows the multiple-shot FEL pulse energy along undulator position (a) and corresponding final spectra (b) for the two-color pulses. The grey lines represent the results of each individual shot and the colored lines represent the average over the 10 shots. The first color 

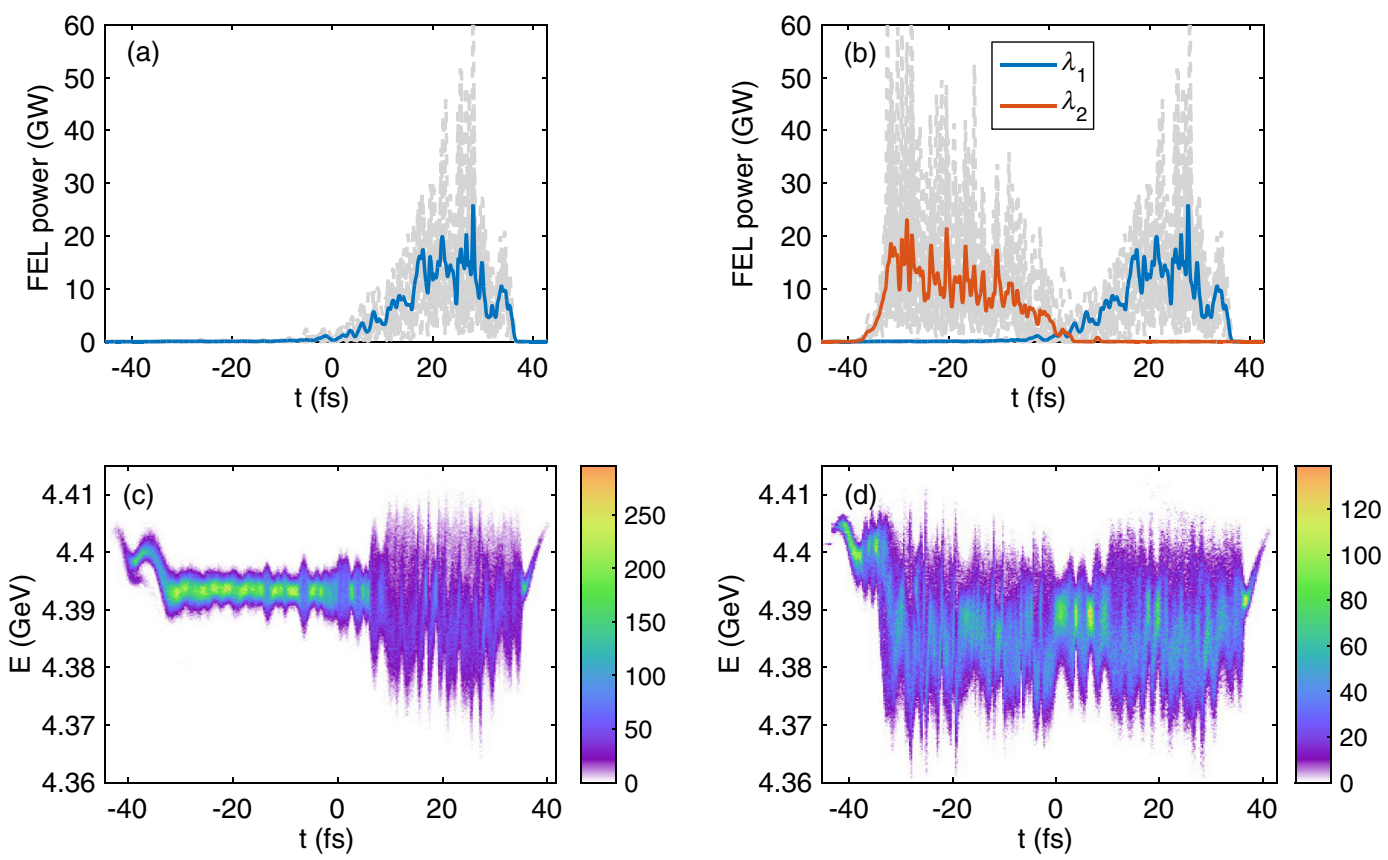

FIG. 11. Multiple-shot FEL power profile (a,b) and single-shot electron bunch longitudinal phase space (c,d) at the end of the first (a,c) and second $(\mathrm{b}, \mathrm{d})$ undulator section. The grey lines represent the results of each individual shot and the colored lines represent the average over the 10 shots. The bunch head is on the left.

at $1.41 \mathrm{~nm}$ wavelength reaches pulse energy of $310 \pm$ $13 \mu \mathrm{J}$ after 7 undulator segments. The second color at $1.45 \mathrm{~nm}$ wavelength reaches pulse energy of $377 \pm 17 \mu \mathrm{J}$ after 8 undulator segments. Since there are still plenty of undulator segments available in both undulator sections, undulator tapering can be applied to obtain a higher pulse energy. With the LCLS undulator, the wavelength separation here is about $2.8 \%$, which can be further increased by imposing a proper energy chirp along electron bunch. Larger wavelength separation can be reached with a variable gap undulator.

Figure 11 shows the multiple-shot FEL power profile $(\mathrm{a}, \mathrm{b})$ and the single-shot electron bunch's longitudinal phase space $(\mathrm{c}, \mathrm{d})$ at the end of the first $(\mathrm{a}, \mathrm{c})$ and second $(b, d)$ undulator section. The grey lines represent the results of each individual shot and the colored lines represent the average over the 10 shots. In Fig. 11(a), the FEL pulse is located at the bunch tail, with a peak power about $15 \pm 1 \mathrm{GW}$. Lasing from the bunch head is suppressed due to the large beta beat introduced by the mismatch. As is shown in Fig. 11(c), the energy spread of the bunch tail is increased while the bunch head remains fresh. After the matching section between the two undulator sections, the bunch head is matched while the bunch tail is mismatched. In the second undulator section, the bunch head lases at the second color, reaching the same level of peak power as the first color pulse, as is shown in Fig. 11(b) and Fig. 11(d). For both color pulses, the power level of the bunch center is lower than that of the tail or head due to residual mismatch in the bunch center. With the magnetic chicane turned off, there is a time delay of about $44 \pm 2$ fs (core to core) between the two pulses. With the magnetic chicane turned on, the time delay of the two-color pulses can be controlled to a large extent or fully cancelled without affecting the pulse duration and energy. The FWHM of the pulse durations for the first and second color are approximately $20 \pm 2 \mathrm{fs}$ and $31 \pm 4 \mathrm{fs}$, respectively. The pulse duration for the second color is longer than for the first color, as a result of the quadratic shape of the mismatch along the bunch. Comparing the FEL pulse duration with the mismatch curves in Figs. 9(b) and 9(d), it can be inferred that a mismatch value about 3 is required to suppress lasing for the LCLS soft X-ray near $1.5 \mathrm{~nm}$ wavelength.

\section{DISCUSSION AND CONCLUSION}

The matching section in the proposed scheme consists of four dedicated matching quadrupoles. Extending the matching section to five quadrupoles can be beneficial. As an underconstrained system, the solution set of the matching problem becomes a one-dimensional curve in the space spanned by the quadrupole strengths, providing more solutions than the four-quadrupole configuration. With one extra quadrupole, the matching section will be able to match larger mismatch back, which helps generate shorter pulse duration and provides more control of the lasing position. For example, it is possible to lase at the bunch center while suppressing both the bunch head and tail from lasing, hence making a three-color configuration possible. For the LCLS, there is an additional quadrupole between 
the horizontal dechirper and the first four-quadrupole matching section, making the five-quadrupole configuration easy to achieve. As for the second matching section, the extra quadrupole can be the quadrupole between the last bending magnet of the chicane and the next undulator segments. Unlike other matching algorithms that usually converge to only one solution, the deterministic matching algorithm we used in this study can find the entire onedimensional family of solutions in a single run.

The matching method relies on the knowledge of the Twiss parameters of the different temporal slices. Practically, measuring the slice Twiss parameters is challenging, due to the lack of a high-resolution slice emittance measurement capability. However, when the dechirper is retracted, the projected Twiss parameters at the entrance of the dechirper can be readily obtained by measuring projected Twiss parameters with wire scanners existing at several locations in the beamline and propagating the measured Twiss from a wire scanner to the dechirper entrance. When the dechirper is inserted, one can use the analytical dechirper model described in Sec. III with either a flat-top current profile or the measured current profile to retrieve the slice Twiss parameters and mismatch. Solving the matching problem for the mismatched target slice gives the quadrupole solution, which would bring the largely mismatched slice close to being matched.

Although the simulation here is for soft x-rays, the proposed method can also be used in the hard x-ray regime. The required mismatch to suppress lasing would be smaller for hard x-rays, but it is also harder to generate and to match back a large mismatch operating at higher beam energy with given maximum quadrupole strength. Hard x-ray, twocolor pulse generation also requires a longer undulator length to reach saturation for each pulse.

In summary, we have formulated and studied in detail a scheme for generating two-color x-ray pulses using the time-dependent focusing effect of the dechirper. The timedependent mismatch generated by the dechirper can be manipulated by matching sections. Taking the LCLS parameters as an example, we show that two-color pulses at $1.41 \mathrm{~nm}$ and $1.45 \mathrm{~nm}$, with independently adjustable delay and pulse duration, can be generated. The scheme takes full advantage of the split undulator-based, twocolor FEL configuration and further increases the FEL power by an effective suppression of unintended lasing. The electron bunch traverses the dechirper on-axis and no dipole kick is introduced, thus avoiding distortion to the beam orbit in the transport system as well as in the undulator, and allowing the method to be applied in high repetition-rate XFELs. Other advanced applications utilizing such a matching-based method may include the amplification of a seed pulse in a self-seeding scheme [29], x-ray pulse duration control, seeding a helical undulator for polarization control [30] and so on. Experimental studies have been planned at the LCLS.

\section{ACKNOWLEDGMENTS}

We thank Z. Huang, K. Bane, T. Liu and K. X. Liu for fruitful discussions. This work is supported by the U.S. Department of Energy under Contract No. DE-AC0276 SF00515.

[1] P. Emma et al., First lasing and operation of an ångstromwavelength free-electron laser, Nat. Photonics 4, 641 (2010).

[2] T. Ishikawa et al., A compact x-ray free-electron laser emitting in the sub-angstrom region, Nat. Photonics 6, 540 (2012).

[3] M. Altarelli et al., Technical Design Report No. DESY 06097, 2007.

[4] K. R. Ferguson et al., Transient lattice contraction in the solid-to-plasma transition, Sci. Adv. 2, e1500837 (2016).

[5] A. Picón et al., Hetero-site-specific x-ray pump-probe spectroscopy for femtosecond intramolecular dynamics, Nat. Commun. 7, 11652 (2016).

[6] A. A. Lutman, R. Coffee, Y. Ding, Z. Huang, J. Krzywinski, T. Maxwell, M. Messerschmidt, and H.D. Nuhn, Experimental Demonstration of Femtosecond Two-Color X-Ray Free-Electron Lasers, Phys. Rev. Lett. 110, 134801 (2013).

[7] T. Hara et al., Two-colour hard x-ray free-electron laser with wide tunability, Nat. Commun. 4, 2919 (2013).

[8] A. Marinelli et al., High-intensity double-pulse x-ray freeelectron laser, Nat. Commun. 6, 6369 (2015).

[9] E. Allaria et al., Two-colour pump-probe experiments with a twin-pulse-seed extreme ultraviolet free-electron laser, Nat. Commun. 4, 2476 (2013).

[10] A. A. Lutman et al., Demonstration of Single-Crystal Self-Seeded Two-Color X-Ray Free-Electron Lasers, Phys. Rev. Lett. 113, 254801 (2014).

[11] E. Ferrari et al., Widely tunable two-colour seeded freeelectron laser source for resonant-pump resonant-probe magnetic scattering, Nat. Commun. 7, 10343 (2016).

[12] V. Kimberg et al., Stimulated x-ray raman scatteringa critical assessment of the building block of nonlinear X-ray spectroscopy, Faraday Discuss. 194, 305 (2016).

[13] G. K. Shenoy, J. W. Lewellen, D. Shu, and N. A. Vinokurov, Variable-period undulators as synchrotron radiation sources, J. Synchrotron Radiat. 10, 205 (2003).

[14] T. Tanaka and H. Kitamura, Composite period undulator to improve the wavelength tunability of free electron lasers, Phys. Rev. ST Accel. Beams 14, 050701 (2011).

[15] A. A. Lutman et al., Fresh-slice multicolour x-ray freeelectron lasers, Nat. Photonics 10, 745 (2016).

[16] K. Bane and G. Stupakov, Corrugated pipe as a beam dechirper, Nucl. Instrum. Methods Phys. Res., Sect. A 690, 106 (2012).

[17] Z. Zhang, K. Bane, Y. Ding, Z. Huang, R. Iverson, T. Maxwell, G. Stupakov, and L. Wang, Electron beam energy chirp control with a rectangular corrugated structure at the Linac Coherent Light Source, Phys. Rev. ST Accel. Beams 18, 010702 (2015). 
[18] M. W. Guetg et al., in Proceedings of 7th International Particle Accelerator Conference (Jacow, Busan, 2016), p. 809.

[19] Y. Chao, Report No. SLAC-PUB-16935, 2016; arXiv:1704 .08813 .

[20] M. W. Guetg, P. J. Emma, M. Santana Leitner, J. J. Welch, and F. Zhou, in Proceedings of 7th International Particle Accelerator Conference (Jacow, Busan, 2016), p. 3755.

[21] D. Ratner et al., Experimental Demonstration of a Soft X-Ray Self-Seeded Free-Electron Laser, Phys. Rev. Lett. 114, 054801 (2015).

[22] J. Amann et al., Demonstration of self-seeding in a hardX-ray free-electron laser, Nat. Photonics 6, 693-698 (2012).

[23] K. Bane, G. Stupakov, and I. Zagorodnov, Analytical formulas for short bunch wakes in a flat dechirper, Phys. Rev. Accel. Beams 19, 084401 (2016).

[24] F. J. Decker, C. Adolphsen, W. J. Corbett, P. Emma, I. Hsu, H. Moshammer, J. T. Seeman, and W. L. Spence, in Proceedings of 1991 IEEE Particle Accelerator Conference (IEEE, San Francisco, 1991), p. 905, Vol. 2.
[25] M. Borland, Advanced Photon Source Report No. LS-287, 2000.

[26] Y. Ding et al., Beam shaping to improve the free-electron laser performance at the Linac Coherent Light Source, Phys. Rev. Accel. Beams 19, 100703 (2016).

[27] K. L.F. Bane et al., Measurements and modeling of coherent synchrotron radiation and its impact on the linac coherent light source electron beam, Phys. Rev. ST Accel. Beams 12, 030704 (2009).

[28] S. Reiche, GENESIS 1.3: a fully 3D time-dependent FEL simulation code, Nucl. Instrum. Methods Phys. Res., Sect. A 429, 243 (1999).

[29] C. Emma, A. A. Lutman, M. W. Guetg, J. Krzywinski, A. Marinelli, J. Wu, and C. Pellegrini, Experimental demonstration of fresh bunch self-seeding in an x-ray free electron laser, Appl. Phys. Lett. 110, 154101 (2017).

[30] A. A. Lutman et al., Polarization control in an x-ray freeelectron laser, Nat. Photonics 10, 468 (2016). 